FORMATION Formation emploi

Revue française de sciences sociales

121 | janvier-mars 2013

L'emploi public : nouveau système de règles, nouvelles stratégies d'acteurs

\title{
Le recrutement des gendarmes adjoints volontaires, un exercice sous tension
}

Recruitment of police assistants in the French Gendarmerie: A difficult exercise Die Einstellung von Polizeiassistenten in der französischen Gendarmerie - eine schwierige Aufgabe

El reclutamiento de los gendarmes adjuntos, un ejercicio bajo tensión

\section{Sébastien Jakubowski}

\section{OpenEdition Journals}

\section{Édition électronique}

URL : http://journals.openedition.org/formationemploi/3907

DOI : 10.4000/formationemploi.3907

ISSN : 2107-0946

\section{Éditeur}

La Documentation française

\section{Édition imprimée}

Date de publication : 15 février 2013

Pagination : 17-37

ISSN : 0759-6340

Référence électronique

Sébastien Jakubowski, « Le recrutement des gendarmes adjoints volontaires, un exercice sous tension », Formation emploi [En ligne], 121 | janvier-mars 2013, mis en ligne le 15 février 2015, consulté le 30 octobre 2020. URL : http://journals.openedition.org/formationemploi/3907 ; DOI : https:// doi.org/10.4000/formationemploi.3907 


\title{
Le recrutement des gendarmes adjoints volontaires, un exercice sous tension
}

\author{
SÉBASTIEN JAKUBOWSKI \\ Maître de conférences en sociologie \\ AgroSup Dijon / Eduter Recherche \\ Chercheur associé au Centre lillois d'études et de recherches sociologiques et économiques \\ (CLERSÉ, université Lille I/CNRS)
}

Résumé

Le recrutement des gendarmes adjoints volontaires, un exercice sous tension

La gendarmerie nationale peine actuellement à recruter. Pour la catégorie étudiée, les gendarmes adjoints volontaires, et en tendance sur la décennie 2000, le nombre de postes offerts au recrutement tout comme le nombre de candidatures ont diminué. Toutefois, depuis 2010, la gendarmerie a rehaussé le nombre de postes disponibles sans parvenir à atteindre un volume suffisant de candidatures recevables, selon ses critères. Ainsi le recrutement, enjeu stratégique pour l'institution, pose problème ; cette situation résulte en partie de l'évolution du métier de gendarme et des transformations de l'institution qui rendent cette activité moins lisible.

Mots clés : Fonction publique, recrutement, armée, gendarme, identité professionnelle

Abstract

Recruitment of police assistants in the French Gendarmerie: A difficult exercise

The French Gendarmerie is currently finding it very difficult to recruit. As far as police assistants are concerned, the study shows that the number of jobs offershad fallen simultaneously with the number of candidates throughout the 2000s. However, since 2010, the French Gendarmerie has raised the number of job offers but failed to reach a sufficient volume of candidates meeting the required criteria. Recruitment, which is a strategic issue for the institution, has become a problem. This is partly the result of an evolution in the exercise of the job and the outcome of institutional changes, which have made this profession less readable for others.

Key words: Civil service, recruitment, armed forces, gendarme, professional identity Journal of Economic Literature: H 83, M 51 
"On entre dans la gendarmerie comme on entre dans les ordres" nous confiait un gendarme il y a quelques années, désireux sans doute de mettre l'accent sur la dimension « vocationnelle » du métier et de l'exercice de l'activité de gendarme.

La gendarmerie, institution régalienne que les français connaissent finalement peu (Dieu, 2008), rencontre aujourd'hui des difficultés de recrutement. Elle se trouve dans une situation paradoxale dans la mesure où elle offre un nombre d'emplois en hausse ces deux dernières années et qu'elle peine à trouver des candidats " recevables " selon ses critères qui sont de deux ordres. Les premiers sont objectifs (jouir de ses droits civiques et être de bonne "moralité ", avoir 17 ans révolus et moins de 26 ans à la date de dépôt de la candidature, être reconnu apte physiquement par un médecin servant dans la gendarmerie, réussir les épreuves de sélection et suivre la formation en école avant l'affectation) ; les seconds critères sont plus subjectifs (principalement la "militarité " : capacité à s'adapter au cadre militaire et conformité avec les valeurs de disponibilité, de service,...). Cette situation paradoxale du recrutement soulève une double question. La première est le positionnement des emplois offerts par les institutions de sécurité (armées, justice, pompiers, police, gendarmerie) sur le marché du travail. La seconde question est celle du modèle républicain d'ascension sociale par les institutions de l'État (Maurin, 2009). Nous verrons ainsi que les postes de gendarmes adjoints volontaires (GAV) sont davantage brigués par des individus issus de la catégorie sociale des ouvriers / employés. En outre, la gendarmerie cherche à séduire de nouveaux candidats en leur réservant des places dans le concours de recrutement des sous-officiers.

Le cas de la gendarmerie est intéressant car cette institution est confrontée à une triple logique : une logique de désinstitutionnalisation (Dubet, 2002), une logique de convergence vers un modèle plus organisationnel et entrepreneurial (Giraud et alii, 2004 ; Padioleau, 2002 ; Moskos, 1987), enfin une logique de recomposition de son cadre institutionnel (Lourau, 1970). La combinaison de ces trois processus affecte les motifs d'engagement des militaires. À défaut d'une vocation réelle, on ne s'engageait pas par hasard, ni vraiment par défaut en gendarmerie. Qu'en est-il aujourd'hui, dans cette institution, de l'exercice du métier de gendarme ? N'est-ce pas devenu un emploi / métier comme un autre ? Les difficultés de recrutement trouvent-elles leur origine dans l'évolution du cadre institutionnel avec le rapprochement entre la gendarmerie et la police nationale ? En quoi et comment l'évolution d'un cadre institutionnel a-t-elle une incidence sur l'exercice d'une profession et sur le regard porté depuis l'extérieur sur celle-ci ?

En octobre 2011, la gendarmerie annonce vouloir recruter 10000 gendarmes dont 7000 gendarmes adjoints volontaires (GAV) et 2500 sous-officiers (SOG). Nous nous intéresserons ici au recrutement des gendarmes adjoints volontaires, catégorie de personnels la plus affectée par les problèmes de recrutement. 
Dans une première partie, nous identifierons la nature du problème du recrutement d'un point de vue sociologique avant de pointer les récentes mesures prises par l'institution pour tenter de pallier ces difficultés. Dans une seconde partie, nous verrons que ces difficultés de recrutement procèdent également pour partie de la rationalisation en cours et d'un changement de configuration "politique » de l'institution.

\section{Encadré 1 \\ Méthodologie de recueil}

Les données utilisées ici ont été recueillies dans le cadre de la coordination d'une mission d'études confiée par un général d'une région de gendarmerie et appuyée par des personnels de la gendarmerie. Ont été remis au groupe de travail un ensemble de statistiques sur le recrutement dans la gendarmerie, établies par la Direction générale de la gendarmerie nationale (DGGN), par l'état-major de cette région, par le Centre d'information et de recrutement (CIR) de celle-ci et par un Centre de sélection et de concours (CSC) de la gendarmerie.

Cet ensemble de données statistiques a été complété par des entretiens et par des observations. Ces dernières ont été réalisées dans un CIR et dans un CSC. Elles ont porté sur des séquences d'accueil dans un CIR conduisant à la remise d'un dossier de recrutement, à des séquences d'entretien de « recrutement », plus exactement de saisie de dossiers de recrutement (en présence des candidats), à l'observation du déroulement des épreuves (écrites et sportives) aux postes de gendarmes adjoints volontaires (GAV) et de sous-officiers de la gendarmerie (SOG), à l'observation d'entretiens de recrutement (auprès d'un officier recruteur) de candidats sous-officiers dans le cadre de l'étude du fonctionnement d'un CSC.

Ces données ont été complétées par des entretiens avec des officiers en poste au sein de la Direction générale de la gendarmerie (par téléphone) et par des entretiens avec des officiers psychologues de la gendarmerie (face à face). Par ailleurs, deux réunions de coordination ont été organisées par l'institution, permettant ainsi des échanges d'informations, de données et d'analyses. Ces éléments ont été recueillis au cours de mai à septembre 2011.

Au total, le corpus de données est constitué de plusieurs jours d'observation, de nombreuses heures de réunion, d'une vingtaine d'entretiens avec des gendarmes occupant plusieurs positions professionnelles. Les questionnements ont principalement porté sur le rapport à l'institution, la carrière, leur expérience de recrutement ou de management des GAV, leur sentiment quant à la reconnaissance (interne et externe) de cette catégorie de personnels, les changements récents dans la gendarmerie. Ces données ont été analysées de façon thématique.

Enfin, ce qui précède ne saurait faire oublier que les données ont été recueillies sous un contrôle institutionnel, c'est-à-dire par un accès limité au terrain et que l'auteur ne peut rendre compte ici de toutes les données. Cet article n'engage évidemment que son auteur. 


\section{Le recrutement des gendarmes adjoints volontaires par une institution militaire}

La gendarmerie nationale, dont la très grande majorité des personnels est sous statut militaire, est une organisation hiérarchique. Pour bien comprendre sa politique de recrutement, la prise en compte de cette caractéristique militaire est d'importance, d'autant que l'institution a évolué au cours de ces dernières années, sous l'effet de la professionnalisation des forces armées, décidée en 1996.

\subsection{Des effectifs en hausse, notamment pour l'encadrement}

La gendarmerie est une institution dont l'ossature est très majoritairement composée de sous-officiers. C'est là une originalité importante par rapport aux autres armées. Toutefois, la professionnalisation des armées est venue modifier la structuration hiérarchique. En effet, la gendarmerie est, d'une part, la seule institution militaire dont les effectifs ont augmenté avec la professionnalisation; elle est, d'autre part, fortement concernée par une mobilité interne qui a permis d'accroître la part des effectifs des officiers et des gendarmes adjoints volontaires (ex militaires du rang et appelés) entre 1997 et 2008. Ce « repyramidage » est intéressant car il s'opère à rebours de celui, par exemple, de l'armée de terre qui cherche, au contraire, à réduire la part de l'encadrement, notamment supérieur ${ }^{1}$.

Tableau 1

Les effectifs militaires et civils de la gendarmerie nationale en $2008\left(^{*}\right)$ et en $1997\left({ }^{* *}\right)$

\begin{tabular}{|c|c|c|c|c|c|}
\hline & & \multicolumn{2}{|c|}{2008} & \multicolumn{2}{|c|}{1997} \\
\hline Affectation des personnels & Catégorie hiérarchique & Effectifs & $\%$ & Effectifs & $\%$ \\
\hline Personnels affectés dans la gendarmerie & & 101134 & $100 \%$ & 94131 & $100 \%$ \\
\hline & Officiers & $6714(1)$ & $6.6 \%$ & 3015 & $3.2 \%$ \\
\hline & Sous-officiers & $78101(2)$ & $77.2 \%$ & 78044 & $82.9 \%$ \\
\hline & Gendarmes adjoints volontaires & 14391 & $14.2 \%$ & 11791 & $12.5 \%$ \\
\hline & Personnels civils & 1928 & $2 \%$ & 1281 & $1.4 \%$ \\
\hline TOTAL & & $105021(3)$ & & 94131 & \\
\hline
\end{tabular}

(*) Source : site internet de la gendarmerie nationale consulté le 3 mai 2011.

$\left({ }^{* *}\right)$ Source : ministère de la Défense, 1998, pp. 7-8-9.

(1) : Dont 239 officiers des corps techniques et de soutien et 25 officiers des autres armées

(2) : Dont 4038 sous-officiers des corps techniques et de soutien.

(3) : On compte également, en 2008, 3887 personnels non affectés dans la gendarmerie. Ces personnels sont affectés dans d'autres services des ministères de la Défense ou de l'Intérieur.

1. Blog de Jean Guisnel, journaliste spécialiste des questions de défense en date du 6 septembre 2012 : http://www.lepoint.fr/chroniqueurs-du-point/jean-guisnel/un-tiers-des-tableaux-d-avancement-supprimespour-2013-06-09-2012-1503186_53.php ; blog de Jean-Dominique Merchet spécialiste des questions de défense en date du 11 septembre 2012 : http://www.marianne.net/blogsecretdefense/Avancements-bloquesle-ministre-de-la-defense-est-tres-tres-embarrasse-_a743.html. 
Dans les années 2000, la principale priorité de la politique de recrutement de la gendarmerie nationale a donc été de pourvoir les postes, en augmentation, des gendarmes adjoints volontaires après la suspension du service national. Le recrutement des volontaires monte en puissance à partir de l'année 1998. Cet enjeu est d'importance pour l'institution dont les missions sont par ailleurs demeurées relativement stables.

Précisons enfin que les caractéristiques singulières des activités de la gendarmerie (missions de police, contacts avec la population civile,...) empêchent de comparer efficacement cette institution avec les trois autres armées (terre, air, marine). À titre d'exemple, l'armée de terre compte dans ses effectifs $52 \%$ d'engagés volontaires qui sont tous, comme les gendarmes adjoints volontaires, des personnels contractuels. Proportionnellement, la gendarmerie compte donc moins de personnels contractuels que de personnels de carrière ou appelés à l'être - ce qui est le cas des sous-officiers et officiers de gendarmerie - que les autres armées.

\section{Encadré 2 \\ Organisation et missions}

La gendarmerie est principalement structurée en deux grandes subdivisions : la gendarmerie départementale, la gendarmerie mobile et quelques autres plus spécialisées (gendarmerie maritime, de l'air, de l'armement,...).

Les forces mobiles participent à l'exécution des missions de sécurité générale en renfort du dispositif territorial. L'unité élémentaire d'organisation de la gendarmerie mobile est l'escadron (certains sont spécialisés : les blindés par exemple). Ils sont répartis sur l'ensemble du territoire national. Des unités spécialisées telles que la garde républicaine ou le groupe d'intervention de la gendarmerie nationale (GIGN) dépendent de la gendarmerie mobile.

La gendarmerie départementale assure, par les brigades de gendarmerie réparties sur l'ensemble du département, des missions de police, de renseignement, de police judiciaire, de contrôle routier,... La gendarmerie départementale constitue l'ossature de l'institution. Les brigades sont principalement situées dans les zones rurales ou périurbaines des départements. Existent des brigades spécialisées : prévention de la délinquance juvénile, sécurité routière, pelotons d'autoroute, unités de montagne,... Les brigades sont organisées en communautés de brigades, groupements (département) et région administrative. Si les missions sont comparables, la gendarmerie départementale se distingue principalement de la police nationale par son implantation géographique et par son organisation du travail au sein des brigades (organisation des horaires de travail, astreintes, vie en caserne,...). 


\subsection{Un recrutement sans conditions de diplôme mais sous statut précaire}

Les gendarmes adjoints volontaires agents de police judiciaire adjoints (GAV APJA) constituent la grande majorité des besoins en recrutement dans la catégorie des GAV ${ }^{2}$. Ils représentent 87,7\% des gendarmes adjoints volontaires recrutés en 2010 .

Ces postes s'adressent à de jeunes français âgés entre 17 et 26 ans. Il n'y a pas de conditions de diplômes. Ces jeunes doivent être aptes physiquement, même si cette aptitude n'est pas contrôlée pendant le processus de recrutement ; elle ne l'est qu'à l'incorporation en école. Les épreuves de sélection se font sur QCM : test psychotechnique, questionnaire de connaissances générales et épreuve de compréhension de texte. Les jeunes candidats obtiennent les résultats jusqu'à deux mois après les épreuves. S’ils sont sélectionnés, ils intègrent pour treize semaines l'une des quatre écoles de gendarmerie (Châteaulin, Chaumont, Montluçon, Tulle). Ils signent un premier contrat d'une durée de deux années, qu'ils peuvent ensuite prolonger par un second contrat d'une durée de trois années. Ils ne peuvent conserver ce statut au-delà de cinq années de service. Ils perçoivent une solde de $878 €$ nets pour un célibataire. Le logement est gratuit et ils peuvent percevoir en fonction de leur affectation à la sortie de l'école une allocation complémentaire d'alimentation de $217 €^{3}$.

Les GAV sont des gendarmes précaires à la durée contractuelle limitée alors que les sousofficiers et officiers peuvent faire carrière dans l'institution. Fidèle à la politique des institutions de l'État, la gendarmerie entend faire des postes de GAV une première porte d'entrée dans l'institution. L'objectif que se donne celle-ci est de conduire $50 \%$ de ses GAV à devenir sous-officiers de la gendarmerie.

\section{Encadré 3 \\ Les activités}

Les GAV sont placés sous l'autorité des sous-officiers de gendarmerie. Affectés en brigade de gendarmerie la plupart du temps, les GAV APJA participent aux activités quotidiennes de contrôle et de surveillance générale mais, n'étant pas et ne pouvant pas être officiers de police judiciaire, leur participation aux procédures est forcément limitée. Ils secondent les sous-officiers dans la plupart des missions dont ils ont la charge : assistance, secours, interventions lors d'accidents ou d'opérations, enquêtes, prévention de la délinquance. De plus, les GAV participent, comme les autres gendarmes, à l'entretien des locaux et des casernements.

2. Les autres spécialités des GAV sont : les aspirants de gendarmerie issus du volontariat (AGIV) qui sont officiers et les emplois particuliers (GAV EP). Les GAV EP sont recrutés pour occuper un poste administratif ou technique dans les domaines du secrétariat, de la restauration, du bâtiment, de la mécanique, de l'horticulture, de l'informatique...

3. La gendarmerie évalue à $217 €$ par mois le montant gratuit de l'hébergement et de l'alimentation qu'elle propose aux GAV. Lorsqu'elle ne peut assurer cela, la rémunération est donc augmentée d'autant. 


\subsection{La mesure statistique des difficultés de recrutement}

Le recrutement dans la gendarmerie est un véritable enjeu institutionnel, aujourd'hui érigé en priorité stratégique (Cavallier, 2011) de l'institution (pour l'année 2011 notamment). La situation dégradée et préoccupante des recrutements des gendarmes adjoints volontaires est celle qui pose le plus question à l'institution.

\subsubsection{Un nombre de postes qui augmente et des candidats qui se raréfient}

Le problème du recrutement des GAV se nourrit de deux phénomènes croisés qui peuvent, selon les interprétations, s'alimenter l'un l'autre. Il y a en effet combinaison d'une augmentation du nombre des postes offerts (au cours de ces deux dernières années et après une période de baisse les années précédentes) et une diminution du nombre de candidats.

Graphique 1 : Evolution du nombre des candidats et des recrutés GAV

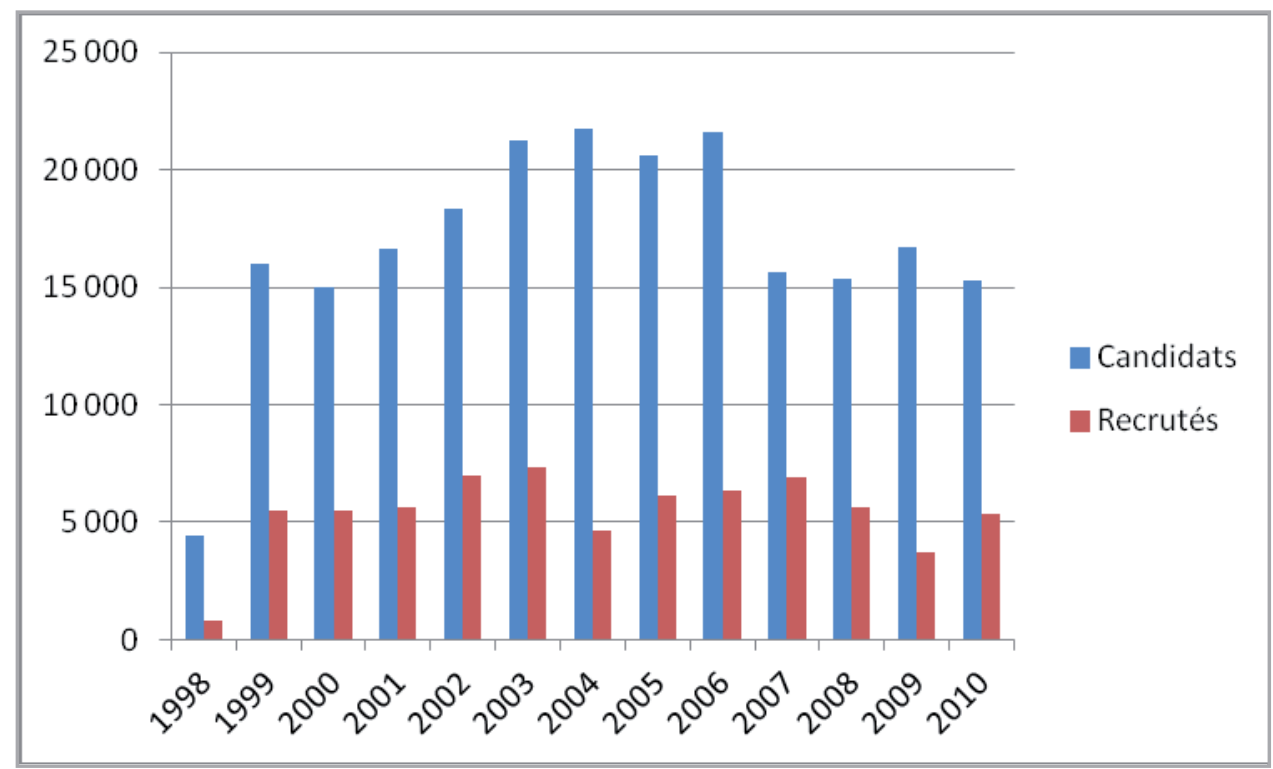

Source: DGGN, 2011, p. 170.

Gav : gendarme adjoint volontaire.

Le nombre des candidats chute à partir de l'année 2006. Entre l'année la plus haute (2004) et l'année la plus basse (2010), le nombre des candidats diminue de $30 \%$.

Lévolution du nombre des recrutés est assez fluctuant. Il augmente fortement entre 1998 et 2003. Il chute fortement en 2004 avant d'augmenter à nouveau jusqu'en 2007. À partir de 2007, le volume des recrutés enregistre une nouvelle baisse. Entre l'année la plus haute (2003) et l'année la plus basse (2009), le nombre des recrutés baisse de près de la moitié. 
Il augmente à nouveau en 2010, année qui marque le retour à une politique de recrutement volontariste.

Mécaniquement, parce qu'il n'y a pas une adéquation et une corrélation entre l'offre et la demande, et parce que l'institution cherche à pourvoir tous ses postes, le taux de sélectivité (DGGN, 2011, p. 170) a connu des évolutions très irrégulières. Au plus haut (en 1998), il a été de 5.5 candidats pour un recruté. Au plus bas (en 2007), il se situe à 2.3 candidats pour un recruté.

\section{Graphique 2 : Taux de sélectivité des GAV}

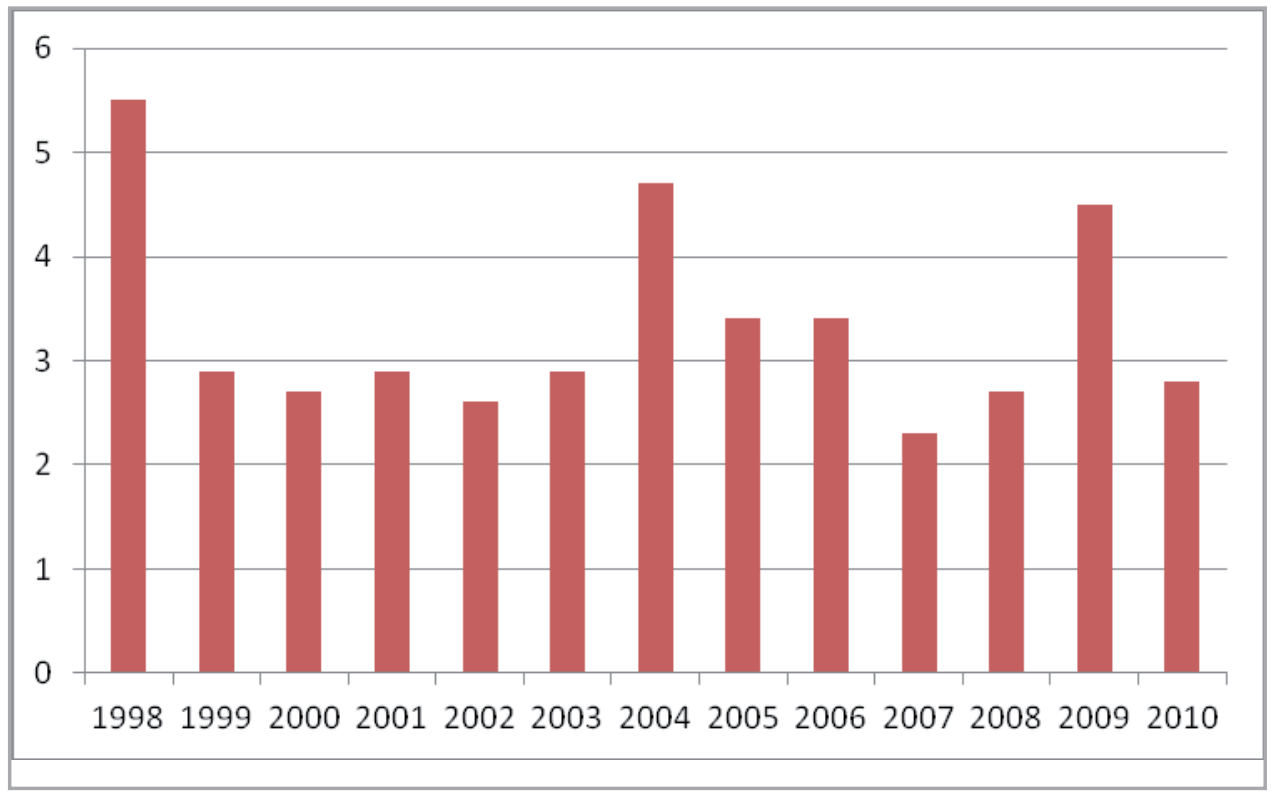

Source : DGGN, 2011, p. 170.

Gav : gendarme adjoint volontaire.

Le problème se pose donc en ces termes. La gendarmerie voit le nombre de ses candidats GAV diminuer de façon significative depuis le milieu des années 2000 alors qu'elle enclenche, à partir de 2010, une politique de recrutement plus conséquente dans cette catégorie de personnels. De plus, dans une lettre qu'elle adresse aux commandants de région, la Direction de la gendarmerie indique qu'elle souhaite un taux de sélectivité (qui lui semble convenable) de 3.5 candidats pour un poste. Cela signifie donc qu'il faut trouver des candidats en masse pour enrayer la baisse, assurer les besoins et maintenir la sélectivité.

En effet, la Direction de la gendarmerie a repéré que le cœur de sa cible des candidats GAV était une population de jeunes françaises et français entre 18 et 26 ans, ce qui représente environ 7000000 de personnes sur le territoire national. Avec 7000 postes offerts pour l'année 2011 et un taux de sélectivité de 3.5, la gendarmerie attend de ses services de recrutement et de ses brigades 24500 dossiers de candidature pour l'année 2011. 


\subsubsection{De nombreuses défections}

Ce que ces chiffres ne disent pas, c'est la forte érosion qui existe à toutes les étapes du recrutement et qui fait perdre à l'institution de l'énergie dans l'emploi de ses ressources. Sur 100 candidats qui remplissent un dossier de candidature, seuls $77 \%$ se présentent effectivement aux épreuves de sélection (DGGN, 2011, p. 195). Parmi eux, $64 \%$ réussissent les épreuves écrites. Parmi ces candidats retenus, $36 \%$ ne rejoignent jamais leur école de formation. Enfin, parmi ceux qui rejoindront leur école, $23 \%$ la quitteront avant la fin de la formation de treize semaines, et $4 \%$ dans les trois mois suivants. À toutes les étapes du recrutement puis de la formation, l'érosion des effectifs dans cette catégorie de personnels est forte.

Tous ces éléments accroissent la préoccupation de la gendarmerie. Cela signifie en effet que sur 100 candidats qui remplissent un dossier de candidature, 23 seront effectivement recrutés et encore présents dans l'institution six mois après leur sortie de l'école 4 . Si l'on accepte un taux de défection et de sélection global effectif de $77 \%$ entre le dossier de candidature et l'arrivée en brigade, cela signifie en projection qu'il faudrait recueillir non pas 24500 mais plutôt 106522 dossiers de candidature. Evidemment, ces calculs ne sont que statistiques et ne prennent pas en compte l'auto-sélection que ces candidats opèrent en ne se présentant pas aux différentes étapes du processus de recrutement.

\subsubsection{Des candidats peu diplômés, issus des milieux employés/ouvriers}

Qui sont les individus qui postulent pour entrer en gendarmerie en tant que GAV ? Nous nous appuierons principalement sur le cas des GAV APJA, catégorie pour laquelle nous disposons des statistiques les plus détaillées et précises.

Tableau 2

Répartition des candidats et des recrutés GAV APJA par sexe et par âge, en 2010

\begin{tabular}{|l|c|c|c|c|c|c|}
\hline & \multicolumn{4}{|c|}{ Candidats } & \multicolumn{3}{c|}{ Recrutés } \\
\hline & Effectifs & $\%$ & Age moyen & Effectifs & $\%$ & Age moyen \\
\hline Hommes & 8922 & 69 & 20 ans & 3357 & 71 & 20 ans et 5 mois \\
\hline Femmes & 4015 & 31 & 19 ans 11 mois & 1348 & 29 & 20 ans et 4 mois \\
\hline Ensemble & 12937 & 100 & 20 ans & 4705 & 100 & 20 ans et 5 mois \\
\hline
\end{tabular}

Source : DGGN, 2011, p. 191 et p. 199.

GAV - APJA : gendarmes adjoints volontaires agents de police judiciaire adjoints.

4. $23=100$ candidats $* 77 \% * 64 \% * 64 \% * 77 \% * 96 \%$. 
La très grande majorité des candidats GAV sont des hommes, ce qui n'est guère étonnant compte tenu de la nature des activités demandées (qui sont physiques et peuvent être risquées) et du niveau général de féminisation des forces armées ou des institutions de sécurité, bien en deçà de la plupart des institutions publiques.

On notera que l'âge moyen des recrutés est légèrement plus élevé que celui des candidats et que les hommes sont plus nombreux en proportion. Ils ont de meilleures chances de réussite.

Tableau 3

Répartition des candidats et des recrutés APJA (par sexe et par niveau d'études) en $\mathbf{2 0 1 0}$

\begin{tabular}{|c|c|c|c|c|c|c|c|c|c|c|c|c|}
\hline & \multicolumn{9}{|c|}{ Candidats } & \multicolumn{5}{c|}{ Recrutés } \\
\hline & \multicolumn{2}{|c|}{ Hommes } & \multicolumn{2}{|c|}{ Femmes } & \multicolumn{2}{c|}{ Ensemble } & \multicolumn{2}{c|}{ Hommes } & \multicolumn{2}{c|}{ Femmes } & \multicolumn{2}{c|}{ Ensemble } \\
\hline $\begin{array}{c}\text { Total Bac } \\
\text { et + }\end{array}$ & 3466 & 46.7 & 1680 & 49.8 & 5146 & 47.6 & 1786 & 58.7 & 720 & 59 & 2506 & 58.8 \\
\hline $\begin{array}{c}\text { Bac+3 } \\
\text { et plus }\end{array}$ & 93 & 1.3 & 61 & 1.8 & 154 & 1.4 & 61 & 2 & 29 & 2.4 & 90 & 2.1 \\
\hline Bac+2 & 266 & 3.6 & 110 & 3.3 & 376 & 3.5 & 159 & 5.2 & 43 & 3.5 & 202 & 4.7 \\
\hline $\begin{array}{c}\text { Bac } \\
\text { général }\end{array}$ & 3107 & 41.8 & 1509 & 44.7 & 4616 & 42.7 & 1566 & 51.5 & 648 & 53.1 & 2214 & 52 \\
\hline $\begin{array}{c}\text { Total } \\
<\text { au Bac }\end{array}$ & 3959 & 53.3 & 1696 & 50.2 & 5655 & 52.4 & 1255 & 41.3 & 500 & 41 & 1755 & 41.2 \\
\hline $\begin{array}{c}\text { Bepc, Bep, } \\
\text { Cap }\end{array}$ & 3907 & 52.6 & 1675 & 49.6 & 5582 & 51.7 & 1246 & 41 & 497 & 40.7 & 1743 & 41 \\
\hline $\begin{array}{c}\text { Sans } \\
\text { diplôme }\end{array}$ & 52 & 0.7 & 21 & 0.6 & 73 & 0.7 & 9 & 0.3 & 3 & 0.3 & 12 & 0.2 \\
\hline Total & $\mathbf{7 4 2 5}$ & $\mathbf{1 0 0}$ & $\mathbf{3 3 7 6} \mathbf{3 7 6}$ & $\mathbf{1 0 0}$ & $\mathbf{1 0 8 0 1}$ & $\mathbf{1 0 0}$ & $\mathbf{3 0 4 1}$ & $\mathbf{1 0 0}$ & $\mathbf{1 2 2 0}$ & $\mathbf{1 0 0}$ & $\mathbf{4 2 6 1}$ & $\mathbf{1 0 0}$ \\
\hline
\end{tabular}

Source : DGGN, 2011, p. 192 et p. 200.

Sigles : APJA : agents de police judiciaires adjoints ; BEPC : brevet des collèges ; BEP : brevet d'études professionnelles ; CAP : certificat d'aptitude professionnelle.

Exemple de lecture : En 2010, $46.7 \%$ des candidats hommes au concours GAV étaient au moins titulaires d'un baccalauréat et $59 \%$ des femmes recrutées étaient titulaires d'un baccalauréat ou plus. Parmi l'ensemble des recrutés (hommes et femmes), $41.2 \%$ étaient titulaires d'un diplôme inférieur au baccalauréat ou ne possédaient pas de diplôme.

Note : N'ont été ici retenus que les effectifs pour lesquels le diplôme est connu. Les effectifs pour lesquels le diplôme n'est pas renseigné ne sont pourtant pas négligeables. Nous pouvons d'ailleurs émettre l'hypothèse que les non-diplômés sont les plus nombreux dans la catégorie « diplôme non renseigné» :

\begin{tabular}{|lccccccc|}
\hline & \multicolumn{3}{c}{ Candidats } & \multicolumn{3}{c|}{ Recrutés } \\
& Hommes & Femmes & Ensemble & Hommes & Femmes & Ensemble \\
Diplôme non renseigné & 1497 & 639 & 2136 & 316 & 128 & 444 \\
\hline
\end{tabular}

Les femmes candidates apparaissent comme étant légèrement plus diplômées que les hommes. Cependant, dans l'ensemble, les candidats n’ont que très rarement un diplôme supérieur au baccalauréat général.

La réussite au concours de recrutement est plus grande lorsque le diplôme est plus élevé. Les recrutés qui ont un niveau au moins égal au baccalauréat général sont plus représentés qu'ils ne le sont parmi les candidats : $52 \%$ des candidats GAV APJA ne pos- 
sèdent pas le baccalauréat contre $41 \%$ des recrutés. L'absence de diplôme semble même constituer un obstacle important au recrutement. Ce résultat est important car la catégorie des GAV APJA s'adresse, initialement, à des individus faiblement ou pas qualifiés.

Tableau 4

Origine socioprofessionnelle du chef de famille des candidats et des recrutés APJA en 2010

\begin{tabular}{|l|c|c|c|c|}
\hline & \multicolumn{2}{|c|}{ Candidats } & \multicolumn{2}{c|}{ Recrutés } \\
\hline & Effectifs & $\%$ & Effectifs & $\%$ \\
\hline Agriculteurs, artisans/commerçants, artistes & 1176 & 10.5 & 443 & 10.5 \\
\hline Employés/ouvriers & 5298 & 47.5 & 1917 & 45.6 \\
\hline Fonctionnaires & 879 & 7.9 & 377 & 9 \\
\hline Cadres, chefs d'entreprise, professions libérales & 1053 & 9.5 & 483 & 11.5 \\
\hline $\begin{array}{l}\text { Militaires de la gendarmerie, militaires } \\
\text { Terre/Air/Mer), Police/douanes, pompiers }\end{array}$ & 609 & 5.5 & 325 & 7.7 \\
\hline Retraités & 1067 & 9.6 & 378 & 9 \\
\hline Sans emploi & 1057 & 9.5 & 279 & 6.7 \\
\hline Total & 11139 & 100 & 4202 & 100 \\
\hline
\end{tabular}

Source : DGGN, 2011, p. 194 et p. 201.

APJA : agents de police judiciaires adjoints.

Note: Non renseignés : Candidats : 1798 ; recrutés : 503.

Deux enseignements principaux peuvent être retirés du tableau 4 quant à l'origine sociale des candidats. Le premier est la très forte proportion de candidats issus d'un milieu social d'employés /ouvriers. Le second enseignement est la très faible représentation, parmi les candidats, de fils et de filles issu-e-s d'un milieu militaire (y compris gendarmerie) ou des institutions de sécurité (pompiers, police, douanes). C’est sans doute l'indicateur le plus inquiétant pour la gendarmerie et pour les gendarmes dont les enfants ne candidatent pas. Peut-être y a-t-il là, en creux, un manque manifeste d'intérêt pour ces postes de la part des gendarmes eux-mêmes. Il s'agit là d'une hypothèse que nous pouvons formuler et qui expliquerait pour partie les difficultés de recrutement.

Il y a finalement peu d'écart d'origine sociale entre les candidats APJA et les recrutés APJA. La catégorie sociale des ouvriers / employés est ainsi la plus représentée. Sans doute est-ce conforme aux attentes pour ce type de postes peu qualifiés qui constituent en réalité une première expérience professionnelle. De manière tout à fait classique, on peut noter que l'origine sociale (supérieure) a un effet positif sur la réussite au recrutement.

\subsection{Les mesures récentes prises par la gendarmerie}

Pour tenter de répondre à cette problématique du recrutement, la gendarmerie adopte une série de mesures. Un rappel s'impose ici quant à l'organisation du dispositif de recrutement de la gendarmerie. Un jeune qui serait intéressé par une carrière ou une expérience professionnelle en gendarmerie doit remplir un dossier de candidature. Il peut le faire dans la brigade de gendarmerie la plus proche de son domicile ou le déposer dans le Centre 
d'informations et de recrutement ${ }^{5}$ (CIR) de la région dans laquelle il réside. Une fois le dossier rempli et déposé (ce qui donne lieu à un premier entretien avec un gendarme de brigade ou un gendarme recruteur), le jeune sera convoqué dans un Centre de sélection et de concours ${ }^{6}$ (CSC) pour y passer les épreuves de sélection.

Les mesures récemment prises par la gendarmerie sont les suivantes :

La première mesure est de nature structurelle. Le processus de recrutement sera davantage décentralisé et géré plus directement au niveau de sept CSC créés à cette occasion sur le territoire national.

La deuxième mesure est une tentative d'introduction de la culture du résultat. D'une part, les CIR des régions sont mis en concurrence, l'objectif étant de faire remplir le plus de dossiers de candidature possible. D'autre part, dans une même région, l'institution met en concurrence les brigades qui doivent remplir $60 \%$ des dossiers et les CIR qui doivent en remplir $40 \%$. Des primes sont attribuées en fonction de la réalisation de ces objectifs.

La troisième mesure est la réforme de l'organisation du concours de recrutement des sousofficiers de gendarmerie. Auparavant " au fil de l'eau ", ce concours est désormais national avec une seule session annuelle ${ }^{7}$. Le but est de rapprocher les modalités de recrutement en gendarmerie de celles en vigueur dans la police nationale (un concours annuel pour le recrutement des gardiens de la paix par exemple). Cette réforme concerne indirectement le recrutement des GAV puisque l'institution réserve $40 \%$ des places au concours sousofficiers à des GAV en activité. L'objectif est de rendre le recrutement GAV plus attrayant par les possibilités de carrière élargies que ce statut peut offrir.

La quatrième mesure met l'accent sur les campagnes de communication. En effet, la gendarmerie a pu constater l'impact immédiat opéré par une campagne nationale de communication sur l'augmentation du nombre des dossiers de candidature. Cependant, on constate que si l'effet des campagnes de publicité est immédiat et intense sur l'enregistrement des dossiers de candidature, il demeure aussi très ponctuel.

Deux campagnes de communication nationale sont intervenues en septembre 2010 et mai 2011. À partir des statistiques disponibles, on repère bien l'effet d'entrainement de ces campagnes sur les dossiers de candidature remplis (Tableau 5).

5. Un CIR est présent dans chaque région administrative française. Les gendarmes recruteurs qui y sont affectés réalisent les missions de communication de la gendarmerie : présence dans les salons et forums, permanences dans des mairies, actions locales de communication. Un CIR dispose d'un budget propre permettant de prendre quelques initiatives ponctuelles mais la stratégie de communication est directement gérée par la direction générale parisienne.

6. Un CSC est bien un centre d'administration des épreuves de sélection. Un CSC est en mesure de sélectionner les candidats par l'échec mais pas par la réussite. En effet, les décisions finales de recrutement sont prises lors d'arbitrages réalisés par la Direction générale parisienne.

7. La première session s'est tenue le 8 février 2012. 
Tableau 5

Evolution du nombre de candidatures avant / après une campagne de communication dans un CIR (Centre d'informations et de recrutement)

\begin{tabular}{|l|c|c|c|c|}
\hline & \multicolumn{2}{|c|}{ Année 2010 } & \multicolumn{2}{c|}{ Année 2011 } \\
\hline & $\mathbf{8}$ premiers mois & $\mathbf{4}$ derniers mois & $\mathbf{4}$ premiers mois & $\mathbf{5}^{\text {ième }}$ mois \\
\hline Moyenne mensuelle & 96 & 154 & 89 & 241 \\
\hline
\end{tabular}

\section{Une institution qui se rationalise et qui change de ministère}

La gendarmerie s'est transformée avec l'introduction de variables plus organisationnelles dans son fonctionnement. Cela s'est traduit par un changement de ministère de tutelle et par des incertitudes sur l'identité professionnelle du gendarme. Ces changements s'accompagnent d'une évolution des motifs de l'engagement dans les institutions militaires professionnalisées.

\subsection{S'engager pour avoir un emploi plutôt que par vocation}

La gendarmerie est désormais rattachée au ministère de l'Intérieur, et elle était déjà l' " armée » la plus " professionnalisée » avant la réforme ; cependant, la comparaison avec les armées plutôt qu'avec la police semble plus probante. D'une part, le statut militaire auquel sont attachés les gendarmes incite à ce rapprochement. D'autre part, les gendarmes eux-mêmes ne se comparent pas aux policiers mais plutôt aux activités et aux métiers d'urgence, plutôt dans le secteur médical ou social puis dans celui de la sécurité (les pompiers) (Giraud et alii, 2004).

En 2003, Jean-François Léger a montré la variété des types d'engagement des jeunes au sein des armées, dans le contexte de la professionnalisation des métiers. Il distinguait les " carriéristes » qui s'engagent pour servir, les "idéalistes » qui s’engagent pour un idéal souvent déçu, les " opportunistes " qui s’engagent pour un métier et les " réfugiés " qui s'engagent pour expérimenter. Outre qu'elle pointe la variété des types d'engagement, cette typologie montre que l'engagement "vocationnel ", loin d'être le plus important en volume, cède aujourd'hui clairement le pas devant un engagement " professionnel " (Jakubowski, Weber, 2011).

Or, et c'est peut-être ici un point de divergence entre cette typologie construite à propos du recrutement des engagés volontaires dans l'armée de terre et le recrutement des GAV en gendarmerie, les individus qui candidatent aux postes de GAV sont, semble-t-il, moins à la recherche d'un engagement professionnel que d'un emploi. 


\section{Encadré 4}

\section{Extrait d'un entretien d'un candidat GAV (gendarme adjoint volontaire) en CIR (Centre d'informations et de recrutement)}

Situation d'observation d'un entretien de motivation (remplissage du dossier de candidature) entre une gendarme recruteuse en CIR (Centre d'informations et de recrutement) et un jeune homme de 22 ans. Ce jeune homme possède un baccalauréat professionnel hygiène et environnement. II n'a pas le permis de conduire, il est célibataire. Il est demandeur d'emploi mais a une expérience de cuisinier/serveur en restauration. Son père est ouvrier d'usine retraité. Sa mère ne travaille pas. Il n'a pas de famille dans l'armée. Voici un extrait de l'entretien.

Gendarme : "Pourquoi candidater en gendarmerie? »

Candidat : " J'aime bien le sport même si je n'en fais pas en ce moment. »

$G$ : «Quels sont les domaines de la gendarmerie qui vous intéressent ?»

$C$ : « C'est trop vague, je ne sais pas. J'attends de voir la période en école. »

$G$ : «Que faites-vous de votre temps libre?»

$C$ : « Je passe mon temps libre à aider mes parents qui ont beaucoup d'ennuis de santé. Je les aide aux tâches ménagères. »

Ce jeune homme ne sera pas en mesure d'expliciter les raisons qui l'ont incité à présenter un dossier de candidature GAV. L'entretien entre la gendarme et le candidat se poursuit alors par une série de questions de culture générale sur les grandes dates clés de l'histoire de France : 8 mai, 14 juillet, 11 novembre,... auxquelles le candidat peine à répondre.

Les évolutions sociales des motifs de l'engagement dans la gendarmerie permettent d'amorcer une réflexion plus large sur l'engagement et la participation des individus en organisation ainsi que le fait Claude Giraud (2011). Dans son introduction, l'auteur montre notamment que le point commun entre l'engagement dans une relation amoureuse et l'engagement dans les armées de métier est la capacité de l'individu à se projeter dans le temps. Or, les candidats sont, par le statut de GAV (qui limite à cinq années la durée maximale du contrat), quelque peu empêchés dans cette projection, sauf à envisager le recrutement GAV comme une étape avant l'accession au corps des sous-officiers.

\subsection{La rationalisation de l'institution ou le glissement vers une logique organisationnelle}

À l'instar d'autres institutions publiques de l'État, la gendarmerie doit rationaliser son organisation, sa gestion des ressources humaines, ses dépenses. Cela crée une pression sur les effectifs et des restructurations importantes dans l'organisation territoriale de la gendarmerie : des escadrons de gendarmerie mobile ont fermé, des brigades territoriales ont 
abandonné certains villages, des services sont regroupés au niveau de l'état-major régional qui lui-même connaît d'importantes restructurations ${ }^{8}$.

Les forts besoins en recrutement de GAV exprimés par la gendarmerie (Gend'info, 2011) s'inscrivent dans la réponse aux besoins du terrain (Crossonneau, 2011). Certes, la population des GAV entrante ne compense pas en volume le nombre des départs de gendarmes ${ }^{9}$; cependant, elle permet un glissement vers une population plus précaire et moins onéreuse pour l'institution ; en effet, son coût de formation est moindre (trois mois de formation contre une année pour les sous-officiers), Il en va de mêmes des charges de personnel ; cette population reste moins longtemps dans l'institution ${ }^{10}$ qui peut la tester et n'en retenir que les meilleurs pour devenir sous-officiers (donc moins de risques sur l'avenir); enfin, cette population peut être encadrée par un seul sous-officier.

C'est donc bien une logique budgétaire qui semble donc primer sur la logique de métier. Cette logique budgétaire fait faire parfois à l'institution un grand écart, comme par exemple entre les consignes données aux gendarmes, en 2009, pour ne pas faire la promotion du métier et l'année 2011 où de nombreuses incitations (y compris des primes pour les meilleures unités) sont introduites pour encourager le recrutement et la promotion du métier.

Ces éléments traduisent, de fait, un glissement d'une logique institutionnelle vers une logique organisationnelle telle qu'elle se repère dans les armées (Jakubowski, 2007 ; Jakubowski, 2011), chez les sapeurs-pompiers (Padioleau, 2002) ou à l'hôpital (Dubet, 2002 ; Herreros, 2007). Sans entrer ici dans le débat théorique sur les définitions des concepts d'institution et d'organisation, ces auteurs s'accordent pour pointer la confrontation entre deux types de rationalité à l'occasion de ce glissement vers une logique organisationnelle. Alors que l'institution se caractérise par la notion de service, un lien social de type communautaire et des valeurs érigées en principe d'action (Douglas, 2004), les réformes organisationnelles introduisent dans l'institution des indicateurs de performance et d'évaluation de l'action ainsi qu'une logique gestionnaire qui contribuent à modifier le lien social, les pratiques sociales et l'identité professionnelle, voire sociale, du métier (Padioleau, 2002).

8. Par exemple, tel état-major qui comprend 195 militaires en octobre 2011 doit réduire ses effectifs de telle sorte qu'il n'y ait plus que 134 militaires et 29 personnels civils à la rentrée 2013. Les missions demeurent inchangées.

9. La gendarmerie devrait perdre 1300 postes de militaires en 2012 et 700 postes de militaires en 2013 . Tous les types d'emploi sont concernés mais principalement ceux des gendarmes en raison des coûts liés à l'astreinte du logement. À rémunération égale, remplacer un militaire de la gendarmerie par un civil coûte moins cher à l'institution.

10. Dans l'idéal pour l'institution, il faudrait qu'un GAV reste au moins quatre années dans l'institution. A partir de quatre années de service, il peut prétendre à un dispositif de reconversion et la gendarmerie n'a plus à lui payer à sa sortie les indemnités chômage. 
Les incertitudes induites par le glissement institutionnel sont bien exprimées par un officier supérieur :

"Il y a, en quelque sorte, une crise identitaire des gendarmes qui ont, comme moi, entre 15 et 20 ans de service. D'ailleurs, l'augmentation des passages à l'acte suicidaire le démontre. Comment trouver sa place dans cette nouvelle institution? C'est un peu la question que se posent ces gendarmes, que je me pose aussi. Il faut bien dire les choses telles qu'elles sont. Depuis 2007, il y a de la violence institutionnelle. La gendarmerie est fortement instable de l'intérieur. Chez nous, on dit que c'est dî̀ à la visée électoraliste qui fait que l'on doit produire de la stat. sur la délinquance, qui fait aussi que l'on redéploie en unités des gendarmes de terrain pour les remplacer par des corps de soutien, qui fait que l'on transforme des postes de gendarmes en postes civils, qui fait que l'on supprime des postes à l'état-major. Ce sont des changements radicaux et, en interne, le discours devient difficilement audible. En tout cas, ce que je sais, je suis bien placé pour le savoir, c'est que cela produit de la souffrance sur la qualité du travail. Je le vois tous les jours. "

Les contraintes de l'exercice du métier de gendarme (le logement en caserne par nécessité absolue de service), les contraintes de la formation et de l'affectation (à au moins $50 \mathrm{~km}$ du domicile) pour les GAV, les contraintes propres au statut militaire jouent un rôle sur le recrutement des gendarmes. Telle est du moins l'hypothèse que nous pouvons formuler.

À cela, il convient d'ajouter les effets des réformes sur l'institution et, pour les GAV, l'instabilité de l'emploi, les tâches ingrates ${ }^{11}$ dévolues dans les unités aux GAV ainsi que le peu de considération qu'ils en retirent, selon leurs dires. L'évolution du positionnement institutionnel de la gendarmerie au sein de l'appareil d'État est certainement aussi importante.

\subsection{Une institution déstabilisée}

Au cours des dernières années, l'institution gendarmique a connu un glissement institutionnel ministériel qui n'est pas anodin sur le fonctionnement de l'institution, sa culture, son identité et ses pratiques de travail. Sans retracer dans cet article toute l'histoire de la gendarmerie, rappelons néanmoins que cette institution est constituée de militaires chargés d'assurer, sur le territoire français, l'ordre public et la sécurité publique. Ce sont des personnels militaires qui exercent des missions civiles en lien avec la population. $\grave{A}$ ces missions civiles (de la gendarmerie départementale comme de la gendarmerie mobile) s'ajoutent des missions militaires en lien avec les autres forces armées (terre, air, mer). Historiquement, la gendarmerie est rattachée au ministère de la Défense. Elle s’est cepen-

11. Certains GAV éprouvent le sentiment que, en tant qu'adjoints des sous-officiers, ils sont exclusivement cantonnés aux tâches les moins gratifiantes et les moins intéressantes au sein des unités : secrétariat, ménage et entretien, surveillance des unités et des sites de la gendarmerie,... 
dant de plus en plus autonomisée de l'armée de terre au cours des trente dernières années. Les choses se sont cependant fortement accélérées dans la décennie $2000^{12}$.

Le président Chirac frâichement élu en 2002 met en œuvre la loi d'orientation et de programmation pour la sécurité intérieure (loi du 29 août 2002). Cette loi confie au ministère de l'Intérieur la coordination et l'autorité sur toutes les missions de sécurité intérieure, y compris donc celles de la gendarmerie nationale qui devient donc pour "emploi ${ }^{13}$ " rattachée à ce ministère. Nicolas Sarkozy, nommé ministre de l'Intérieur, conduit cette politique. La gendarmerie est alors conduite et contrainte à se réorganiser, ce qui sera acté en 2005.

D'une part, elle crée des communautés de brigades et des brigades territoriales autonomes (BTA). D'autre part, elle se redéploie sur le territoire national avec la police nationale pour tenir compte des mouvements de la population sur le territoire national au cours des dernières années. Enfin, elle harmonise son organisation territoriale par groupements (les départements) et par régions (correspondant aux régions administratives). Depuis cette période, la division historique du territoire entre les deux forces de sécurité intérieure, à savoir les campagnes à la gendarmerie et les villes à la police, n'est sans doute plus aussi juste.

L'année 2009 marque un autre tournant d'importance pour la gendarmerie. Ses budgets (et non plus seulement ses missions) sont gérés par le ministère de l'Intérieur ; la loi du 3 août 2009 intègre la gendarmerie à ce ministère tout en réaffirmant le caractère militaire du statut des gendarmes qui ne sont plus désormais gérés par le ministère de la Défense que pour la composante « emploi » dans le cadre de leurs missions et prérogatives militaires.

Ces réformes institutionnelles et cette double appartenance (la gendarmerie a deux sites internet institutionnels officiels : un pour la défense et un pour l'intérieur) ont déstabilisé fortement l'institution et ses pratiques en la " civilisant » davantage, en rationalisant son organisation et ses procédures, en la confrontant aussi à l'institution policière de manière plus directe ${ }^{14}$. L'identité du métier de gendarme en a été modifiée par ce rapprochement avec la police nationale.

12. La réforme de la gendarmerie s'est accélérée après les « manifestations » des gendarmes au cours de l'hiver 2001. Les revendications principales portaient sur le manque de moyens et les conditions de travail : véhicules hors d'état ou hors d'âge, des astreintes et horaires de service trop éprouvants, le fait de devoir travailler dans les unités avec les équipements informatiques de la famille,...

13. C'est-à-dire que le ministère de l'Intérieur devient, pour les missions civiles (plus de $90 \%$ des missions de la gendarmerie), le donneur d'ordres. Le ministère de la Défense continue, à cette époque encore, à s'occuper de la gestion administrative des personnels et reste (aujourd'hui encore) donneur d'ordres pour les missions militaires de la gendarmerie. De fait, à partir de cette date, la gendarmerie dépend presqu'exclusivement du ministère de l'Intérieur, ce qui constitue une rupture « historique » dans l'organisation de cette institution.

14. Dans le cadre de la participation à une manifestation en préfecture avec des gendarmes, l'auteur a ainsi pu assister à un face à face très tendu entre les deux corps de sécurité. Un policier en tenue refusait l'accès à la préfecture aux gendarmes sous prétexte qu'ils étaient armés. Il a fallu qu'un lieutenant-colonel de gendar- 
C'est d'ailleurs ce qu'admet un autre officier supérieur :

"Il est clair qu'il nous faut maintenant assouplir les conditions d'intégration des GAV en leur donnant des garanties sur le transport, l'éloignement géographique,... ainsi que des informations précises sur le statut militaire. On a un vrai problème avec la mobilité géographique et les contraintes de la discipline militaire chez les GAV. Avant, il y avait le filtre du service national... Les GAV ont pour vocation de répondre aux besoins du terrain : tenir une arme, répondre au stress, contribuer à assurer les plans de charge au sein des unités. Il est clair aussi que nous devons maintenant nous mettre au diapason avec la police nationale. Par exemple, il faudra que $85 \%$ des GAV soient recrutés avec le bac en poche."

L'identité professionnelle du gendarme évolue avec le positionnement institutionnel de la gendarmerie. La gendarmerie du $\mathrm{XXI}^{\circ}$ siècle ne pourra faire l'impasse sur son adaptation à ces évolutions et à la "modernisation" (Luc, 2010). Il est clair que la gendarmerie est aujourd'hui en question (Matelly, 2006) ; d'ailleurs, le sociologue Laurent Muchielli (2012) n'hésite pas à affirmer que les " gendarmes n’ont vraiment pas le moral».

\section{Conclusion}

La gendarmerie nationale rencontre des problèmes de recrutement, en particulier dans la catégorie des gendarmes adjoints volontaires. La focalisation de l'article sur les GAV (gendarmes adjoints volontaires), qui constituent l'enjeu stratégique de l'institution, masque néanmoins d'une part, la complexité de cette institution qui comprend de très nombreux postes spécialisés à tous les niveaux hiérarchiques et d'autre part, la grande hétérogénéité des situations professionnelles exercées et vécues par les gendarmes (Fontaine, 2008). Si les difficultés de recrutement sont moindres pour les postes spécialisés (qui constituent une faible partie du recrutement en gendarmerie), c'est aussi parce que les règles du jeu institutionnel semblent plus limpides. L'individu se positionne sur un poste clairement identifié et auquel est parfois rattaché un lieu d'exercice correspondant à ses qualifications professionnelles. L'armée de terre, par exemple, procède de la sorte. Les jeunes souhaitant la rejoindre sur un poste d'engagé volontaire

merie se déplace pour discuter avec ce policier en tenue puis avec d'autres de ses collègues pour débloquer la situation. Au-delà de l'anecdote, cet épisode illustre des rapports parfois tendus entre les deux institutions. L'adoption de grades de type militaire dans la police nationale engendre un sentiment de dévalorisation parmi les gendarmes : un commandant en gendarmerie par exemple n'a ainsi, selon les gendarmes, pas les mêmes responsabilités, pas les mêmes activités professionnelles, ni le même cursus académique qu'un commandant de police. 
candidatent sur un poste au titre d'un régiment. Si le dispositif de recrutement et d'affectation des GAV manque de clarté, ce n'est sans doute pas la cause principale des difficultés de recrutement.

Comme nous l'avons vu précédemment, les candidats gendarmes adjoints volontaires (GAV) sont principalement issus des catégories sociales employés / ouvriers. À la recherche d'un emploi ou d'une première expérience professionnelle, ils se font certainement aussi une représentation des métiers de la sécurité et de leur exercice au sein d'une institution telle que la gendarmerie nationale. Or, le statut de GAV renvoie à l'occupation d'un poste précaire, faiblement rémunéré, peu valorisé au sein de l'institution car il s'agit principalement de fonctions d'exécution. De plus, les GAV ont parfois le sentiment d'un manque de considération : ils sont, par exemple, les seuls gendarmes à ne pas pouvoir loger en caserne avec leur famille. Ces éléments, parce qu'ils associent d'une certaine façon cette catégorie de personnel au prolétariat (par la précarité, la faible rémunération, les activités et certaines conditions de travail comme de vie), sont certainement à la base des difficultés de recrutement. D'autres causes interviennent également, semble-t-il, comme les changements institutionnels et leurs effets au sein de l'institution. De l'extérieur, ces changements ne sont certes pas immédiatement perceptibles par les candidats potentiels aux postes GAV mais ils ont des incidences fortes chez les gendarmes (Jakubowski, 2006) qui sont les premiers représentants de leur institution.

En effet, la gendarmerie est une institution qui a changé, comme beaucoup d'autres institutions publiques sont en train de changer. Ces institutions sont confrontées au marché d'une part (pas uniquement au marché du travail) et à un rapport nouveau au politique d'autre part. Le glissement institutionnel de la gendarmerie illustre parfaitement à la fois une forme nouvelle de mainmise du politique sur les institutions, accentuée ici par le côté régalien de la gendarmerie, et une certaine logique d'instrumentalisation, par le politique, de la production de ces institutions. Les institutions de sécurité sont encore confrontées au marché de la sécurité. Sur ce marché, de multiples acteurs interviennent (les forces de police, la justice, les sapeurs pompiers, les douanes, la sécurité civile, l'administration fiscale, l'administration pénitentiaire) parfois en complément (les groupes régionaux d'intervention) mais parfois aussi en concurrence (la police judiciaire). À ces acteurs publics s’ajoutent les agences privées de sécurité. Face à ce marché de la sécurité en fort développement et moins transparent qu'autrefois, il semble que la gendarmerie peine actuellement à séduire les jeunes candidats potentiels. Gendarmerie nationale (avec les GAV) et police nationale (avec les adjoints de sécurité (ADS) sont ainsi en concurrence frontale sur le marché du travail. Ce que la professionnalisation des armées a montré, dans ce registre, en France depuis dix ans - à savoir par exemple la nécessité d'une communication professionnelle plus claire -, la gendarmerie est en train de l'expérimenter. Cette situation est paradoxale pour une institution qui vient de quitter la Défense pour rejoindre le giron de l'Intérieur. Mais peut-être cela produira-t-il un nouvel élan... 
Le cas de la gendarmerie nationale apporte un éclairage sociologique pertinent sur les problèmes que rencontre actuellement la fonction publique. Le recrutement joue l'effet d'un miroir grossissant les incertitudes, les contraintes, les changements, les questionnements que rencontrent les professionnels au sein des institutions publiques. En la matière et du point de vue des professions, le nœud des difficultés rencontrées par celles-ci semble être une certaine crise des vocations professionnelles. Ces professions attirent beaucoup moins (métiers de l'enseignement, de la sécurité,...) en partie parce que les contours et le contenu de leur activité ont changé alors que, jusqu'à une période très récente, dans un contexte de crise économique et de chômage, ces professions et ces institutions véhiculaient des valeurs captives depuis l'extérieur : une certaine sécurité de l'emploi, des conditions de travail acceptables, la possibilité de concilier vie professionnelle et personnelle, une solidarité de " corps ». Or, les processus de rationalisation de ces institutions semblent avoir dissous ces modes structurants de solidarité et semblent faire converger le contrat social en œuvre vers un modèle entrepreneurial qui promeut des valeurs différentes. Dans ces conditions, intégrer la fonction publique et ses institutions prend moins de sens. Les candidats intéressés n’hésitent plus à mettre en concurrence tous les employeurs potentiels et à se répartir ainsi sur l'ensemble du marché du travail.

\section{Bibliographie}

Cavallier B. (2011), «Recruter : une priorité stratégique et un défi permanent pour la gendarmerie ", Revue de la gendarmerie nationale, $1^{\text {er }}$ trimestre, pp. 41-50.

Crossonneau M.-O. (2011), "Le recrutement, un défi pour les armées ", Revue Défense Nationale, $\mathrm{n}^{\circ} 741$, juin, pp. 47-55.

Direction Générale de la Gendarmerie Nationale (2011), Eléments statistiques sur le recrutement. Année 2010, DPMGN, Paris.

Dieu F. (2008), Sociologie de la gendarmerie, L'Harmattan, Paris.

Douglas M. (2004), Comment pensent les institutions, La Découverte / Poche, Paris.

Dubet F. (2002), Le Déclin de l'institution, Seuil, Paris.

Fontaine J.-Y. (2007), Socio-anthropologie du gendarme. Gendarmerie et démocratie, L'Harmattan, "Logiques Sociales », Paris.

Gend’info (2011), «2011, année recrutement », n³34, février.

Giraud C. (dir.), Jakubowski S. et alii (2004), De l'institution à l'organisation de la gendarmerie : les effets induits sur le métier de gendarme, Les Documents du C2SD, Paris.

Giraud C. (2011), Qu'est-ce que l'engagement?, L'Harmattan, «Logiques sociales », Paris. 
Herreros G. (2007), «L'hôpital à l'épreuve des réformes ", Socio-anthropologie [en ligne], $\mathrm{n}^{\circ} 21$, mis en ligne le 26 novembre 2008, consulté le 30 septembre 2011.

Jakubowski S., Weber C. (2011), Etre militaire dans l'armée de terre, Lieux-Dits, Lyon.

Jakubowski S. (2011), "L'institution militaire confrontée aux réformes organisationnelles ", Année Sociologique, vol. ${ }^{\circ} 61, \mathrm{n}^{\circ}$ 2, pp. 297-322.

Jakubowski S. (2007), La professionnalisation de l'armée française : conséquences sur l'autorité, L’Harmattan, «Logiques sociales », Paris.

Jakubowski S. (2006), "Les effets des changements organisationnels sur les représentations professionnelles. Le cas de la gendarmerie française ", Studia Europaea, n ${ }^{\circ} 1$, pp. 193-216.

Léger J.-F. (2003), " Pourquoi des jeunes s'engagent-ils aujourd'hui dans les armées ? ", Revue française de sociologie, $\mathrm{n}{ }^{\circ} 44-4$, pp. 713-734.

Lourau R. (1970), L'Analyse institutionnelle, Les Éditions de Minuit, Paris.

Luc J.-N. (dir.) (2010), Soldats de la loi. La gendarmerie au XXe siècle, Presses de l'Université Paris-Sorbonne, Paris.

Matelly J.-H. (2006), Une police judiciaire... militaire ? La gendarmerie en question, L'Harmattan, "Sécurité et société ", Paris.

Maurin E. (2009), La peur du déclassement, Seuil, « La République des idées », Paris.

Ministère de la Défense (1998), Bilan social 1997, Secrétariat général pour l’administration, Paris.

Moskos C. (1987), « La Banalisation de l'institution militaire. L’armée américaine : du modèle institutionnel au modèle industriel ", Futuribles, n ${ }^{\circ} 111$, pp. 27 -37.

Muchielli L. (2012), Vous avez dit sécurité ?, Editions Champ social, Paris.

Padioleau J.-G. (2002), Le Réformisme pervers : le cas des sapeurs-pompiers, PUF, Paris. 